\title{
New form of generlized closed sets via neutrosophic topologicl spaces
}

\author{
BinoyBalan $\mathbf{e K}^{1 *}$, C.Janaki ${ }^{2} \&$ Sandhya P.S ${ }^{3}$ \\ ${ }^{1}$ Department of Science \& Humanities, Ahalia School of Engineering and Technology, Palakkad, Kerala, India \\ ${ }^{2}$ Department of Mathematics, L.R.G. College for Women, Tirupur-4, India \\ ${ }^{3}$ Department of Applied Science \& Humanities, Royal College of Engineering and Technology, Thrissur, Kerala, India \\ Email: binoypayyur@gmail.com ${ }^{1}$,janakicsekar@yahoo.com ${ }^{2} \&$
}

Article History: Received: 11 January 2021; Accepted: 27 February 2021; Published online: 5 April 2021

\begin{abstract}
Florentin eSmarandache egeneralized ethe eintuitionistic efuzzy esets eto eNeutrosophic eset etheory ein e1998 eas ea enew ebranch eof ephilosophy. eA.A. eSalama eintroduced ethe econcept eof eNeutrosophic etopological espaces eby eusing ethe eNeutrosophic ecrisp esets. eIn ethis epaper, ewe eintroduce eand estudy ea enew eclass eof eNeutrosophic egeneralized eset, enamely eNeutrsophic epre egeneralized eregular e $\alpha$ - eclosed eset ein eNeutrosophic etopological espaces. eSome einteresting epropositions ebased eon ethis eset eare eintroduced eand established ewith esuitable examples eand etheir eproperties eare ealso ediscussed.
\end{abstract}

Keywords- $\mathcal{N}$ pgr $\alpha$ - closed set, $\mathcal{N}$ pgr $\alpha$ - open set, $\mathcal{N}$ pgr $\alpha$-closure, $\mathcal{N}$ pgr $\alpha$ - interior

\section{INTRODUCTION AND PRELIMINARIES}

L.A. Zadeh [20] introduced the concept of fuzzy sets in 1965. It shows the degree of membership of the lement in a set. Later, fuzzy topology was introduced by C.L.Chang [6] in 1968. Coker [7] introduced the notion of Intuitionistic fuzzy topological spaces by using Atanassov's [5] Intuitionistic fuzzy set. Neutrality the degree of indeterminacy, as an independent concept, was introduced by Smarandache [19] in 1998. He also defined the Neutrosophic set on three components, namely Truth (membership), Indeterminacy, Falsehood (nonmembership) from the Fuzzy sets and Intuitionistic fuzzy sets. Smarandache's Neutrosophic concepts have wide range of real time applications for the fields of

[[1]-[4],[10],[12],[15],[18]] Information systems, Computer science, Artificial Intelligence, Applied Mathematics and Decision making. A.A. Salama and S.A. Alblowi [16] introduced Neutrosophic topological spaces by using the Neutrosophic sets. Salama A. A. [17] introduced Neutrosophic closed set and Neutrosophic continuous functions in Neutrosophic topological spaces.R.Dhavaseelan and SaiedJafari [8] introduced Neutrosophic generalized closed sets. In this direction, we introduce and analyze a new class of Neutrosophic generalized closed set called Neutrsophic pre generalized regular $\alpha$ - closed set which is the weaker form of the above mentioned generalization and its properties are discussed in details.

Definition 1.[16]Let $\mathcal{J}$ be a non-empty fixed set. A Neutrosophic set [NS for short] $\mathcal{A}$ is an object having the form $\mathcal{A}=\left\{\left\langle\mathrm{a}, \quad \mu_{\mathcal{A}}(\mathrm{a}), \quad \sigma_{\mathcal{A}}(\mathrm{a}), \quad v_{v}(\mathrm{a})\right\rangle: \mathrm{a} \in \mathcal{J}\right\}$ where $\mu_{\mathcal{A}}(\mathrm{a}), \quad \sigma_{\mathcal{A}}(\mathrm{a})$ and $v_{\mathcal{A}}(\mathrm{a})$ which represent the degree of membership function, degree of indeterminacy and the degree of non-membership respectively of each element $\mathrm{x} \in \mathrm{A}$ to the set $\mathcal{A}$.

Remark 1.2. [16] A Neutrosophic set $\mathcal{A}=\left\{\left\langle\mathrm{a}, \mu_{\mathcal{A}}(\mathrm{a}), \sigma_{\mathcal{A}}(\mathrm{a}), v_{\mathcal{A}}(\mathrm{a})\right\rangle:\right.$ a $\left.\in \mathcal{J}\right\}$ can be identified to an ordered triple $\mathcal{A}=\left\langle\mathrm{a}, \mu_{\mathcal{A}}(\mathrm{a}), \sigma_{\mathcal{A}}(\mathrm{a}), v_{\mathcal{A}}(\mathrm{a})\right\rangle$ in non-standard unit interval $]^{-0}, 1^{+}[$on $]$.

For the sake of simplicity, we shall use the $\quad \mathcal{A}=\left\langle\mu_{\mathcal{A}}(\mathrm{a}), \sigma_{\mathcal{A}}(\mathrm{a}), v_{\mathcal{A}}(\mathrm{a})\right\rangle$ for the Neutrosophic set $\mathcal{A}=\left\{\left\langle\mathrm{a}, \mu_{\mathcal{A}}(\mathrm{a}), \sigma_{\mathcal{A}}(\mathrm{a}), v_{v}(\mathrm{a})\right\rangle: \mathrm{a} \in \mathcal{J}\right\}$.

Definition 1.3. [16] Every Intuitionistic fuzzy set $\mathcal{A}$ is

a nonempty set in $\mathcal{J}$ is obviously on Neutrosophic set having the form $\mathcal{A}=\left\{<\mathrm{a}, \mu_{\mathcal{A}}(\mathrm{a}), 1-\left(\mu_{\mathcal{A}}(\mathrm{a})+v_{\mathcal{A}}(\mathrm{a})\right), v_{\mathcal{A}}(\mathrm{a})\right.$ $>: \mathrm{a} \in \mathcal{J}\}$. Since our main purpose is to construct the tools for developing Neutrosophic set and Neutrosophic topology, we must introduce the Neutrosophic set $0_{\mathcal{N}}$ and $1_{\mathcal{N}}$ in $\mathcal{J}$ as follows:

$0_{\mathcal{N}}$ may be defined as:

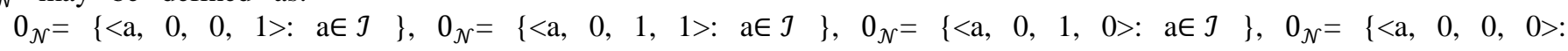
$\mathrm{a} \in \mathcal{J}\}$

$1_{\mathcal{N}}$ may be defined as:

$1_{\mathcal{N}}=\{\langle a, 1,0,0\rangle: a \in \mathcal{J}\}, 1_{\mathcal{N}}=\{\langle a, 1,0,1\rangle: a \in \mathcal{J}\}, 1_{\mathcal{N}}=\{\langle a, 1,1,0\rangle: a \in \mathcal{J}\}, 1_{\mathcal{N}}=\{\langle a, 1, \quad 1,1\rangle: a \in$ $\mathcal{J}\}$

Definition 1.4. [16] Let $\mathcal{A}=<\mu_{\mathcal{A}}(\mathrm{a}), \sigma_{\mathcal{A}}(\mathrm{a}), v_{\mathcal{A}}(\mathrm{a})>$ be an NS on $\mathcal{J}$ then the complement of the set $\mathcal{A}$ $[\mathrm{C}(\mathcal{A})$ for short] may be defined as three kinds of complements:

(i) $\mathrm{C}(\mathcal{A})=\left\{\left\langle\mathrm{a}, 1-\mu_{\mathcal{A}}(\mathrm{a}), 1-\sigma_{\mathcal{A}}(\mathrm{a}), 1-v_{\mathcal{A}}(\mathrm{a})\right\rangle: \mathrm{a} \in \mathcal{J}\right\} \quad$ (ii) $\mathrm{C}(\mathcal{A})=\left\{\left\langle\mathrm{a}, v_{\mathcal{A}}(\mathrm{a}), \sigma_{\mathcal{A}}(\mathrm{a}), \mu_{\mathcal{A}}(\mathrm{a})\right\rangle: \mathrm{a} \in \mathcal{J}\right\}$

(iii) $\mathrm{C}(\mathcal{A})=\left\{\left\langle\mathrm{a}, v_{\mathcal{A}}(\mathrm{a}), 1-\sigma_{\mathcal{A}}(\mathrm{a}), \mu_{\mathcal{A}}(\mathrm{a})>: \mathrm{a} \in \mathcal{J}\right\}\right.$ 
Definition 1.5. [5] Let $\mathcal{J}$ be a nonempty set. Let $\mathcal{S}$ and $\mathcal{T}$ be any Neutrosophic sets on $\mathcal{J}$ in the form $\mathcal{S}=\left\{\left\langle\mathrm{a}, \mu_{\mathcal{S}}(\mathrm{a}), \quad \sigma_{\mathcal{S}}(\mathrm{a}), \quad v_{\mathcal{S}}(\mathrm{a})\right\rangle: \mathrm{a} \in \mathcal{J}\right\}$ and we may consider two possible definitions for subsets $\left.\mathcal{T}=\left\{<\mathrm{a}, \quad \mu_{\mathcal{T}}(\mathrm{a}), \quad \sigma_{\mathcal{T}}(\mathrm{a}), \quad v_{\mathcal{T}}(\mathrm{a})\right\rangle: \mathrm{a} \in \mathcal{J}\right\}$. Then be defined as:

$(\mathcal{S} \subseteq \mathcal{T}) . \mathcal{S} \subseteq \mathcal{T}$ may

$$
\mathcal{S} \subseteq \quad \mathcal{T} \Leftrightarrow \mu_{\mathcal{S}}(\mathrm{x}) \leq \quad \mu_{\mathcal{T}}(\mathrm{x}), \sigma_{\mathcal{S}}(\mathrm{x}) \leq \quad \sigma_{\mathcal{T}}(\mathrm{x}),
$$

$v_{\mathcal{S}}(\mathrm{x}) \geq v_{\mathcal{T}}(\mathrm{x})$ for all $\mathrm{a} \in \mathcal{J}$.

$\mathcal{S} \subseteq \mathcal{T} \Leftrightarrow \mu_{\mathcal{S}}(\mathrm{x}) \leq \quad \mu_{\mathcal{T}}(\mathrm{x}), \sigma_{\mathcal{S}}(\mathrm{x}) \geq \quad \sigma_{\mathcal{T}}(\mathrm{x}), \quad v_{\mathcal{S}}(\mathrm{x}) \geq v_{\mathcal{T}}(\mathrm{x})$ for all a $\in \mathcal{J}$

Proposition 1.6. [5] For any Neutrosophic set $\mathcal{A}$ the following conditions hold: $0_{\mathcal{N}} \subseteq \mathcal{A}, \quad 0_{\mathcal{N}} \subseteq 0_{\mathcal{N}}, \mathcal{A} \subseteq 1_{\mathcal{N}}, 1_{\mathcal{N}} \subseteq 1_{\mathcal{N}}$

Definition 1.7.[16] Let $\mathcal{J}$ be a nonempty set. Let $\mathcal{S}=\left\{\left\langle\mathrm{a}, \mu_{\mathcal{S}}(\mathrm{a}), \sigma_{\mathcal{S}}(\mathrm{a}), v_{\mathcal{S}}(\mathrm{a})\right\rangle\right\}, \mathcal{T}=\left\{\left\langle\mathrm{a}, \mu_{\mathcal{T}}(\mathrm{a}), \sigma_{\mathcal{T}}(\mathrm{a}), v_{\mathcal{T}}(\mathrm{a})\right\rangle\right\}$ are Neutrosophic sets. Then $\mathcal{S} \cap \mathcal{T}$ may be defined as:

(i) $\quad \mathcal{S} \cap \mathcal{T}=<\mathrm{a}, \mu_{\mathcal{S}}(\mathrm{a}) \wedge \mu_{\mathcal{T}}(\mathrm{a}), \sigma_{v}(\mathrm{a}) \wedge \sigma_{\mathcal{T}}(\mathrm{a}), v_{\mathcal{S}}(\mathrm{a}) \vee v_{\mathcal{T}}(\mathrm{a})>$

(ii) $\quad \delta \cap \mathcal{T}=\left\langle\mathrm{a}, \mu_{\mathcal{S}}(\mathrm{a}) \wedge \mu_{\mathcal{T}}(\mathrm{a}), \sigma_{\mathcal{S}}(\mathrm{a}) \vee \sigma_{\mathcal{T}}(\mathrm{a}), v_{\mathcal{S}}(\mathrm{a}) v_{\mathcal{T}}(\mathrm{a})\right\rangle$

$\mathcal{S} \cup \mathcal{T}$ may be defined as:

(i) $\mathcal{S} \cup \mathcal{T}=\left\langle\mathrm{a}, \mu_{\mathcal{S}}(\mathrm{x}) \vee \mu_{\mathcal{T}}(\mathrm{x}), \quad \sigma_{\mathcal{S}}(\mathrm{x}) \vee \sigma_{\mathcal{T}}(\mathrm{x}), \quad v_{\mathcal{S}}(\mathrm{x}) \wedge v_{\mathcal{T}}(\mathrm{x})>\right.$

(ii) $\mathcal{S} \cup \mathcal{T}=\left\langle\mathrm{x}, \mu_{\mathcal{S}}(\mathrm{x}) \vee \mu_{\mathcal{T}}(\mathrm{x}), \sigma_{\mathcal{S}}(\mathrm{x}) \wedge \sigma_{\mathcal{T}}(\mathrm{x}), v_{\mathcal{S}}(\mathrm{x}) \wedge \nu_{\mathcal{T}}(\mathrm{x})\right\rangle$

We can easily generalize the operations of intersection and union to arbitrary family of Neutrosophic sets as follows:

Definition 1.8. [16] Let $\left\{\mathcal{A}_{\mathrm{j}}: \mathrm{j} \in \mathrm{J}\right\}$ be a arbitrary family of Neutrosophic sets in $\mathrm{A}$, then $\cap \mathcal{A}_{\mathrm{j}}$ may be defined as

(i) $\quad \cap \mathcal{A}_{\mathrm{j}}=\left\langle\mathrm{a}, \wedge_{j \in J} \mu_{\mathcal{A}_{j}}(\mathrm{a}), \wedge_{j \in J} \sigma_{\mathcal{A}_{j}}(\mathrm{a}), \mathrm{V}_{j \in J} \nu_{\mathcal{A}_{j}}\right.$ (a)>

(ii) $\cap \cap \mathcal{A}_{j}=\left\langle\mathrm{a}, \wedge_{j \in J} \mu_{\mathcal{A}_{j}}(\mathrm{a}), \mathrm{V}_{j \in J} \sigma_{\mathcal{A}_{j}}(\mathrm{a}), \mathrm{V}_{j \in J} v_{\mathcal{A}_{j}}(\mathrm{a})>\right.$

$\cup \mathcal{A}_{j}$ may be defined as

(i) $\quad \cup \mathcal{A}_{j}=<\mathrm{a}, \quad \mathrm{v}_{j \in J} \mu_{\mathcal{A}_{j}}(\mathrm{a}), \mathrm{v}_{j \in J} \sigma_{\mathcal{A}_{j}}(\mathrm{a}), \wedge_{j \in J} v_{\mathcal{A}_{j}}(\mathrm{a})>$

(ii) $\quad \cup \mathcal{A}_{j}=\left\langle\mathrm{a}, \vee_{j \in J} \mu_{\mathcal{A}_{j}}(\mathrm{a}), \wedge_{j \in J} \sigma_{\mathcal{A}_{j}}(\mathrm{a}), \wedge_{j \in J} \nu_{\mathcal{A}_{j}}\right.$ (a) $>$

Proposition 1.9. [16] For two Neutrosophic sets $\mathcal{S}$ and $\mathcal{T}$, the following conditions are true:

$\mathrm{C}(\mathcal{S} \cap \mathcal{T})=\mathrm{C}(\mathcal{S}) \cup \mathrm{C}(\mathcal{T}) ; \mathrm{C}(\mathcal{S} \cup \mathcal{T})=\mathrm{C}(\mathcal{S}) \cap \mathrm{C}(\mathcal{T})$.

Definition 1.10 [16] A Neutrosophic topology [NT] on a nonempty set $\mathcal{J}$ is a family $\tau$ of Neutrosophic subsets in $\mathcal{J}$ satisfying the following axioms:

(i) $\quad 0_{\mathcal{N}}, 1_{\mathcal{N}} \in \tau$

(ii) $\mathcal{G}_{1} \cap \mathcal{G}_{2} \in \tau$ for any $\mathcal{G}_{1}, \mathcal{G}_{2} \in \tau$

(iii) $\quad \cup \mathcal{G}_{i} \in \tau$ for every $\left\{\mathcal{G}_{i}: \mathrm{i} \in \mathrm{J}\right\} \subseteq \tau$

The pair of $(\mathcal{J}, \tau)$ is called Neutrosophic topological space [NTS for short]. The elements of $\tau$ are called Neutrosophic open set [NOS for short]. A Neutrosophic set $\mathcal{F}$ is Neutrosophic closed set [NCS for short] if and only if $\mathrm{C}(\mathcal{F})$ is Neutrosophic open set.

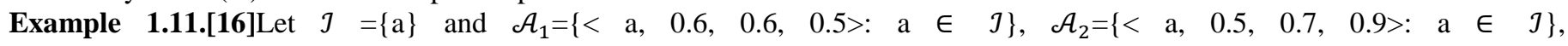

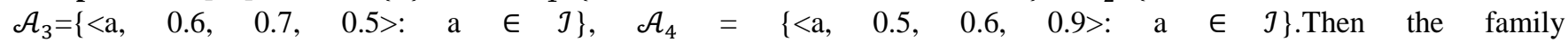
$\tau=\left\{0_{\mathcal{N}}, \mathcal{A}_{1}, \mathcal{A}_{2}, \mathcal{A}_{3}, \mathcal{A}_{4}, 1_{\mathrm{N}}\right\}$ is called a Neutrosophic topological space on $\mathcal{J}$.

Definition 1.12[16] Let $(\mathcal{J}, \tau)$ be an NTS and $\mathcal{A}=\left\langle\mathrm{a}, \quad \mu_{\mathcal{S}}(\mathrm{a}), \quad \sigma_{\mathcal{S}}(\mathrm{a}), \quad v_{\mathcal{S}}(\mathrm{a})\right\rangle$ be an NS in $\mathcal{J}$. Then the Neutrosophic closure and Neutrosophic interior of $\mathcal{A}$ are defined by $\operatorname{NCl}(\mathcal{A})=\cap\{\mathcal{K}: \mathcal{K}$ is an $\mathrm{NCS}$ in $\mathcal{J}$ and $\mathcal{A} \subseteq \mathcal{K}\}, \quad \operatorname{NInt}(\mathcal{A})=\cup\{\mathcal{G}: \mathcal{G}$ is an $\operatorname{NOS}$ in $\mathcal{J}$ and $\mathcal{G} \subseteq \mathcal{A}\}$

It can be also shown that $\operatorname{NCl}(\mathcal{A})$ is $\operatorname{NCS}$ and $\operatorname{Nint}(\mathcal{A})$ is a $\operatorname{NOS}$ in $\mathcal{J}$.

(i) $\quad \mathcal{A}$ is $\mathrm{NOS}$ if and only if $\mathcal{A}=\operatorname{NInt}(\mathcal{A})$

(ii) $\mathcal{A}$ is $\mathrm{NCS}$ if and only if $\mathcal{A}=\operatorname{NCl}(\mathcal{A})$

Proposition 1.13.[16]For any Neutrosophic set $\mathcal{A}$ in $(\mathcal{J}, \tau)$ we have

(i) $\quad \mathrm{NCl}(\mathrm{C}(\mathcal{A}))=\mathrm{C}(\operatorname{NInt}(\mathcal{A}))$

(ii) $\quad \operatorname{NInt}(\mathrm{C}(\mathcal{A}))=\mathrm{C}(\mathrm{NCl}(\mathcal{A}))$

Proposition 1.14.[16] Let $(\mathcal{J}, \tau)$ be a NTS and $\mathcal{S}, \mathcal{T}$ be two Neutrosophic sets in $\mathcal{J}$. Then the following properties hold:

(i) $\quad \operatorname{NInt}(\mathcal{S}) \subseteq \quad \mathcal{S} \subseteq \mathrm{NCl}(\mathcal{S})$

(ii) $\quad \mathcal{S} \subseteq \mathcal{T} \Rightarrow \operatorname{NInt}(\mathcal{S}) \subseteq \operatorname{NInt}(\mathcal{T})$ and $\operatorname{NCl}(\mathcal{S}) \subseteq \mathrm{NCl}(\mathcal{T})$

(iii) $\quad \operatorname{NInt}(\operatorname{NInt}(\mathcal{S}))=\operatorname{NInt}(\mathcal{S})$

(iv) $\quad \mathrm{NCl}(\mathrm{NCl}(\mathcal{S}))=\operatorname{NCl}(\mathcal{S})$

(v) $\quad \operatorname{NInt}(\mathcal{S} \cap \mathcal{T})=\operatorname{NInt}(\mathcal{S}) \cap \operatorname{NInt}(\mathcal{T})$ 
(vi) $\quad \operatorname{NCl}(\mathcal{S} \cup \quad \mathcal{T})=\operatorname{NCl}(\mathcal{S}) \cup \mathrm{NCl}(\mathcal{T})$

(vii) $\operatorname{NInt}\left(0_{\mathcal{N}}\right)=0_{\mathcal{N}}, \operatorname{NInt}\left(1_{\mathcal{N}}\right)=1_{\mathcal{N}}$

(viii) $\mathrm{NCl}\left(0_{\mathcal{N}}\right)=0_{\mathcal{N}}, \operatorname{NCl}\left(1_{\mathcal{N}}\right)=1_{\mathcal{N}}$

(ix) $\quad \mathcal{S} \subseteq \mathcal{T} \Rightarrow \mathrm{C}(\mathcal{S}) \subseteq \mathrm{C}(\mathcal{T})$

(x) $\quad \mathrm{NCl}(\quad \mathcal{S} \cap \quad \mathcal{T}) \subseteq \mathrm{NCl}(\mathcal{S}) \cap \mathrm{NCl}(\mathcal{T})$

(xi) $\quad \operatorname{NInt}(\mathcal{S} \cup \quad \mathcal{T}) \subseteq \operatorname{NInt}(\mathcal{S}) \quad \cup \operatorname{NInt}(\mathcal{T})$

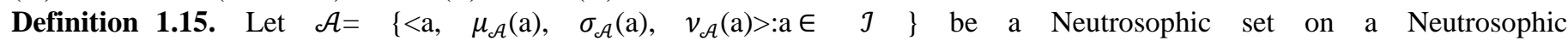
topological space $(\mathcal{J}, \tau)$ then $\mathcal{A}$ is called

(i) Neutrosophic regular open set (NROS for short)[12] if $\mathcal{A}=\operatorname{NInt}(\mathrm{NCl}(\mathcal{A})$ ).

(ii) Neutrosophic pre-open set (NPOS for short)[14] if $\mathcal{A} \subseteq \operatorname{NInt}(\mathrm{NCl}(\mathcal{A})$.

(iii) Neutrosophic $\alpha$-open set $(\mathrm{N} \alpha \mathrm{OS}$ for short)[14] if $\mathcal{A} \subseteq \mathrm{NInt}(\mathrm{NCl}(\mathrm{NInt}(\mathcal{A}))$ ).

An NS $\mathcal{A}$ is called Neutrosophic regular closed set, Neutrosophic pre closed set and Neutrosophic $\alpha$-closed (NRCS, NPCS and N $\alpha$ CS for short) if the complement of $\mathcal{A}$ is NROS, NPOS and N $\alpha$ OS respectively.

Definition 1.16.[13]Let $\mathcal{A}=\left\{<\mathrm{a}, \mu_{\mathcal{A}}(\mathrm{a}), \sigma_{\mathcal{A}}(\mathrm{a})\right.$, $\left.v_{\mathcal{A}}(\mathrm{a})>\mathrm{a} \in \mathcal{J} \quad\right\}$ be a Neutrosophic set on Neutrosophic topological space $(\mathcal{J}, \tau)$. Then the Neutrosophic pre-

closure and Neutrosophic pre interior of $\mathcal{A}$ are defined by $\operatorname{NPCl}(\mathcal{A})=\{\mathcal{K}: \mathrm{K}$ is a NPCS in $\mathcal{J}$ and $\mathcal{A} \subseteq \mathcal{K}\}$,

NPInt $(\mathcal{A})=\{\mathcal{G}: \quad$ is a NPOS in $\mathcal{J}$ and $\subseteq \mathcal{A}\}$

Definition 1.17.[12] Let $\mathcal{A}=\left\{<\mathrm{a}, \mu_{\mathcal{A}}(\mathrm{a}), \sigma_{\mathcal{A}}(\mathrm{a})\right.$, $\left.v_{\mathcal{A}}(\mathrm{a})>: \mathrm{a} \in \mathcal{J}\right\}$ be a Neutrosophic set on a Neutrosophic

topological

space $(\mathcal{J}, \tau)$. Then the Neutrosophic $\alpha$-closure

and Neutrosophic $\alpha$-interior of $\mathcal{A}$ are defined by

$\mathrm{N} \alpha \mathrm{Cl}(\mathcal{A})=\{\mathcal{K}: \mathcal{K}$ is a $\mathrm{N} \alpha \mathrm{CS}$ in $\mathcal{J}$ and $\mathcal{A} \subseteq \mathcal{K}\}$,

$\mathrm{N} \alpha \operatorname{Int}(\mathcal{A})=\{\mathcal{G}: \mathcal{G}$ is a $\mathrm{N} \alpha \mathrm{OS}$ in $\mathcal{J}$ and $\mathcal{G} \subseteq \mathcal{A}\}$

Definition 1.18. Let $\mathcal{A}=\left\{\left\langle\mathrm{a}, \mu_{\mathcal{A}}(\mathrm{a}), \sigma_{\mathcal{A}}(\mathrm{a}), v_{\mathcal{A}}(\mathrm{a})>\mathrm{a} \in \mathcal{J}\right\}\right.$

be a Neutrosophic set on a Neutrosophic topological space

$(\mathcal{J}, \tau)$. Then $\mathcal{A}$ is called

(i) Neutrosophic regular generalized closed set (NRGCS for short)[9], if $\mathrm{NCl}(\mathcal{A}) \subseteq \mathrm{U}$, whenever $\mathcal{A} \subseteq$ $\mathcal{U}$ and $\mathcal{U}$ is a Neutrosophic regular open set in $\mathcal{J}$.

(ii) Neutrosophic regular $\alpha$-generalized closed set (NRaGCS for short)[9], if $\mathrm{N} \alpha \mathrm{Cl}(\mathcal{A}) \subseteq \mathcal{U}$, whenever $\mathcal{A} \subseteq$ $\mathcal{U}$ and $\mathcal{U}$ is a Neutrosophic regular open set in $\mathcal{J}$.

(iii) Neutrosophic generalized pre closed set (NGPCS for short)[13] if $\operatorname{NPCl}(\mathcal{A}) \subseteq \mathcal{U}$ whenever $\mathcal{A} \subseteq U$ and $\mathcal{U}$ is a Neutrosophic open set in $\mathcal{J}$.

(iv) Neutrosophic generalized pre regular closed set (NGPRCS for short)[11] if $\operatorname{NPCl}(\mathcal{A}) \subseteq \mathcal{U}$ whenever $\mathcal{A} \subseteq$ $\mathcal{U}$ and $\mathcal{U}$ is a Neutrosophic regular open set in $\mathcal{J}$.

An NS $\mathcal{A}$ is called Neutrosophic regular generalized open set, Neutrosophic regular $\alpha$-generalized open set, Neutrosophic generalized pre-open set and Neutrosophic generalized pre regular open set (NRGOS,NR $\alpha$ GOS, NGPOS and NGPROS for short) if the complement of $\mathcal{A}$ is NRGCS,NR $\alpha$ GCS, NGPCS and NGPRCS respectively.

\section{NEUTROSOPHIC PRE GENERALIZED REGULAR $\alpha$-CLOSED SET}

In this section, we introduce Neutrosophic pre generalized regular $\alpha$ - closed set and analyze some of their properties.

Definition 2.1. Let $\mathcal{A}=\left\{\left\langle\mathrm{a}, \mu_{\mathcal{A}}(\mathrm{a}), \sigma_{\mathcal{A}}(\mathrm{a}), v_{\mathcal{A}}(\mathrm{a})\right\rangle: \mathrm{a} \in \mathcal{J} \quad\right\}$ be a Neutrosophic set on a Neutrosophic topological space $(\mathcal{J}, \tau)$.Then $\mathcal{A}$ is called Neutrosophic regular $\alpha$-open set (NR $\alpha \mathrm{OS}$ for short) if there is a Neutrosophic regular open set $\mathcal{U}$ such that $\mathcal{U} \subset \mathcal{A} \subset N \alpha \mathrm{Cl}(\mathcal{U})$.A Neutrosophic set $\mathcal{A}$ of a Neutrosophic space $(\mathcal{J}, \tau)$ is called a Neutrosophic regular $\alpha$-closed set (NR $\alpha \mathrm{CS}$ for short) if $\mathrm{C}(\mathcal{A})$ is a $\mathrm{NR} \alpha \mathrm{OS}$ in $(\mathcal{J}, \tau)$.

Definition 2.2. Let $\mathcal{A}=\left\{\left\langle\mathrm{a}, \mu_{\mathcal{A}}(\mathrm{a}), \sigma_{\mathcal{A}}(\mathrm{a}), v_{\mathcal{A}}(\mathrm{a})\right\rangle: \mathrm{a} \in \mathcal{J}\right\}$ be a Neutrosophic set on a Neutrosophic topological space $(\mathcal{J}, \tau)$. Then $\mathcal{A}$ is called Neutrosophic pre generalized regular $\alpha$ - closed set $(\mathcal{N} \mathcal{P} \mathcal{G R} \alpha \mathrm{CS}$ for short), if $\operatorname{NPCl}(\mathcal{A}) \subseteq \mathcal{U}$ whenever $\mathcal{A} \subseteq U$ and $U$ is a $\operatorname{NR} \alpha \mathrm{OS}$ in $(\mathcal{J}, \tau)$.

Alternatively, a Neutrosophic set $\mathcal{A}$ is said to be a Neutrosophic pre generalized regular $\alpha$-open set $(\mathcal{N} \mathcal{P} \mathcal{G} \mathcal{R} \alpha \mathrm{OS}$ for short) if $\mathrm{C}(\mathcal{A})$ is a $\mathcal{N} \mathcal{P} \mathcal{G} \mathcal{R} \alpha \mathrm{CS}$ in $(\mathcal{J}, \tau)$.

The family of all $\mathcal{N} \mathcal{P} \mathcal{G R} \alpha \mathrm{CSs}[\mathcal{N} \mathcal{P} \mathcal{G} \mathcal{R} \alpha \mathrm{OSs}]$ of an NTS $(\mathcal{J}, \tau)$ is denoted by $\mathcal{N} \mathcal{P} \mathcal{G} \mathcal{R} \alpha \mathrm{C}(\mathcal{J}) \quad[\mathcal{N} \mathcal{P} \mathcal{G} \mathcal{R} \alpha \mathrm{O}(\mathcal{J})]$.

Example 2.3. Let $\mathcal{J}=\{\mathrm{a}, \mathrm{b}\}$ and $\tau=\left\{0_{\mathcal{N}}, \mathrm{U}, \mathrm{V}, 1_{\mathcal{N}}\right\}$ where $U=\{\langle 0.5,0.3,0.6\rangle,\langle 0.4,0.4,0.7\rangle\}$ and $\mathcal{V}=\left\{\begin{array}{lll}\langle 0.7,0.5,0.3\rangle,\langle 0.7,0.5,0.2\rangle\end{array}\right.$. Then $(\mathcal{J}, \tau)$ is a Neutrosophic topological space. Here the 
Neutrosophic set $\mathcal{A}=\{\langle 0.5,0.3,0.4\rangle,\langle 0.6,0.6,0.3\rangle\}$ is a $\mathcal{N} \mathcal{P} \mathcal{G} \alpha \operatorname{CS}$ in $(\mathcal{J}, \tau)$. Since $\mathcal{A} \subseteq \mathcal{U}$ and $\mathcal{U}$ is a $\mathrm{NR} \alpha \mathrm{OS}$, we have $\operatorname{NPCl}(\mathcal{A})=\mathcal{A} \subseteq \mathcal{U}$.

\section{Theorem 2.4.}

(i) Every Neutrosophic closed set is $\mathcal{N} \mathcal{P} \mathcal{G} \mathcal{R} \alpha$ - closed set in $\mathcal{J}$.

(ii) Every Neutrosophic regular closed set is $\mathcal{N} \mathcal{P} \mathcal{G} \mathcal{R} \alpha$-closed set in $\mathcal{J}$.

(iii) Every Neutrosophic pre closed set is $\mathcal{N} \mathcal{P} \mathcal{G} \mathcal{R} \alpha$-closed set in $\mathcal{J}$.

(iv) Every Neutrosophic $\alpha$-closed set is $\mathcal{N} \mathcal{P} \mathcal{G} \mathcal{R} \alpha$-closed set in $\mathcal{J}$.

(v) Every $\mathcal{N} \mathcal{P} \mathcal{G} \mathcal{R} \alpha$-closed set is Neutrosophic generalized pre regular closed set in $\mathcal{J}$.

(vi) Every Neutrosophic regular $\alpha$-generalized closed set in $\mathrm{X}$ is a $\mathcal{N} \mathcal{P} \mathcal{G} \mathcal{R} \alpha$-closed set in $\mathcal{J}$.

(vii) Every Neutrosophic generalized pre closed set in $\mathrm{X}$ is a $\mathcal{N} \mathcal{P} \mathcal{G} \mathcal{R} \alpha$-closed set in $\mathcal{J}$.

(viii) Every Neutrosophic regular generalized closed set in $\mathrm{X}$ is a $\mathcal{N} \mathcal{P} \mathcal{G} \mathcal{R} \alpha$-closed set in $\mathcal{J}$.

Proof: Straight forward. Converse of the above need not be true as in the following examples.

Example 2.5.

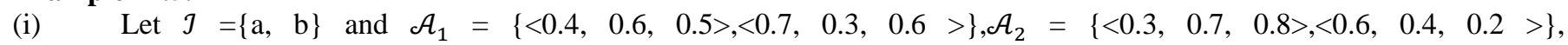

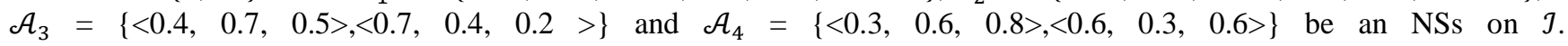
Now $\tau=\left\{0_{\mathcal{N}} \mathcal{A}_{1}, \mathcal{A}_{2}, \mathcal{A}_{3}, \mathcal{A}_{4}, 1_{\mathcal{N}}\right\}$ is a Neutrosophic topological spaces on J.Then $\mathcal{A}=\{\langle 0.3,0.6,0.8\rangle$, $<0.5,0.3,0.7>\}$ is $\mathcal{N} \mathcal{P} \mathcal{G} \mathcal{R} \alpha \mathrm{CS}$ in $\mathcal{J}$. But $\mathcal{A}$ is not NCS, NRCS, NPCS, N $\alpha$ CS in $\mathcal{J}$.

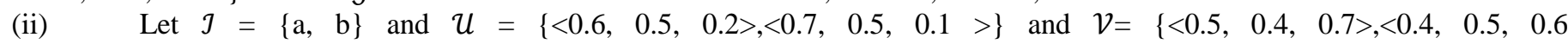
$>\}$, be an NSs on $\mathcal{J}$. Now $\tau=\left\{0_{\mathcal{N}}, 1_{\mathcal{N}}, \mathcal{U}, \mathcal{V}\right\}$ is a Neutrosophic topological spaces on $\mathcal{J}$. Here the Neutrosophic set $\mathcal{A}=\{\langle 0.8,0.6,0.1\rangle,\langle 0.8,0.6,0\rangle\}$ is a Neutrosophic generalized pre regular closed set in J. But $\mathcal{A} \subseteq \mathcal{W}$ and $\mathcal{W}=\{\langle 0.5,0.5,0.8\rangle,\langle 0.4,0.3,0.6\rangle\}$ is $\mathrm{NR} \alpha \mathrm{OS}$ and $\mathrm{NPCl}(\mathrm{A})=1_{\mathcal{N}} \nsubseteq \mathcal{W}, \mathcal{A}$ is not a $\mathcal{N} \mathcal{P} \mathcal{G} \mathcal{R} \alpha$-closed set in $\mathcal{J}$.

(iii) Let $\mathcal{J}=\{\mathrm{a}, \mathrm{b}\}$ and $\mathcal{U}=\{\langle 0.6,0.5,0.2\rangle,\langle 0.7,0.5,0.1\rangle\}$ and $\mathcal{V}=\{\langle 0.5,0.4,0.7\rangle,\langle 0.4,0.5$, $0.6>\}$, be an NSs on $\mathcal{J}$. Now $\tau=\left\{0_{\mathrm{N}}, 1_{\mathrm{N}}, \mathcal{U}, \mathcal{V}\right\}$ is a Neutrosophic topological spaces on J. Here the Neutrosophic set $\mathcal{A}=\{\langle 0.4,0.3,0.7\rangle,\langle 0.3,0.2,0.6\rangle\}$ is a $\mathcal{N} \mathcal{P} \mathcal{G} \mathcal{R} \alpha \mathrm{CS}$ in $\mathcal{J}$. But $\mathcal{A}$ is not

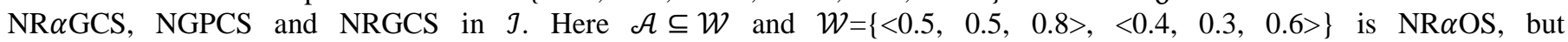
not NOS and NROS in $\mathcal{J}$.

Remark 2.6. The above discussions are summarized in the following diagram.

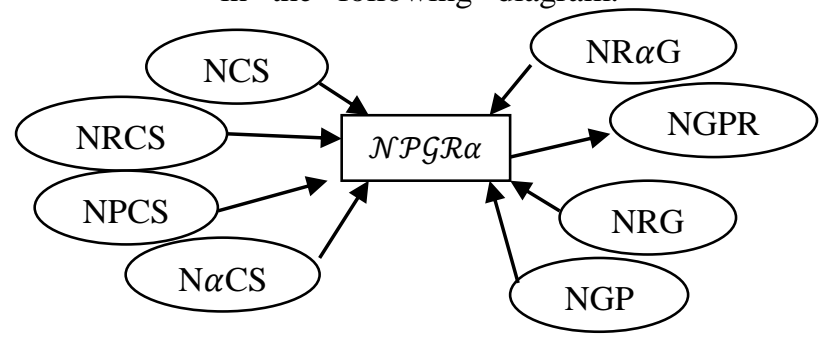

Remark 2.7. The union of any two $\mathcal{N} \mathcal{P} \mathcal{G} \mathcal{R} \alpha \mathrm{CSs}$ in $(\mathcal{J}, \tau)$ is not an $\mathcal{N} \mathcal{P} \mathcal{G} \mathcal{R} \alpha \mathrm{CS}$ in $(\mathcal{J}$, $\tau)$ in general as seen from the following example.

Example 2.8. Let $\mathcal{J}=\{\mathrm{a}, \mathrm{b}\}$ and $\tau=\left\{0_{\mathcal{N}}, 1_{\mathcal{N}}, \mathcal{U}, \mathcal{V}\right\}$ where $\mathcal{U}=\{\langle 0.5,0.3,0.6\rangle,\langle 0.4,0.4,0.7\rangle\}$ and

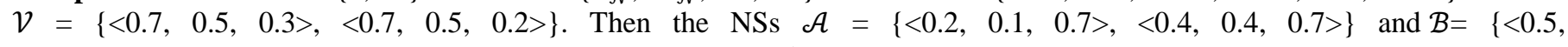

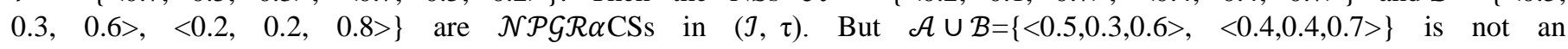
$\mathcal{N} \mathcal{P} \mathcal{G} \mathcal{R} \alpha \mathrm{CS}$ in $(\mathcal{J}, \tau)$.

Since $(\mathcal{A} \cup \mathcal{B}) \subseteq \mathcal{U}$ and $\operatorname{NPCl}(\mathcal{A} \cup \mathcal{B})=\{<0.6,0.7,0.5), \quad(0.7,0.6,0.4>\}=\mathrm{C}(\mathcal{U}) \nsubseteq \mathcal{U}$

Remark 2.9.: The intersection of any two $\mathcal{N} \mathcal{P} \mathcal{G} \mathcal{R} \alpha \mathrm{CSs}$ in

$(\mathcal{J}, \tau)$ is not an $\mathcal{N} \mathcal{P} \mathcal{G} \mathcal{R} \alpha \mathrm{CS}$ in $(\mathcal{J}, \tau)$ in general as seen from the following example.

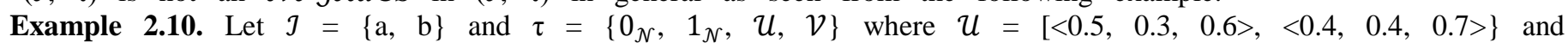

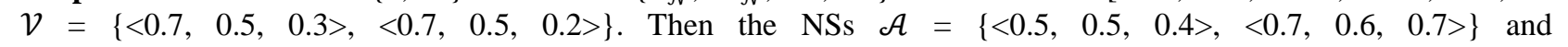

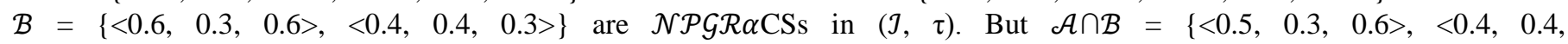

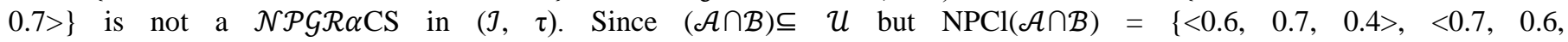
$0.4>\} \nsubseteq \mathcal{U}$.

Theorem 2.11. Let $(\mathcal{J}, \tau)$ be an NTS. Then for every $\mathcal{A} \in \operatorname{NPGR} \alpha \mathrm{C}(\mathcal{J})$ and for every Neutrosophic set $\mathcal{B} \in \mathrm{NS}(\mathcal{J}), \mathcal{A} \subseteq \mathcal{B} \subseteq \mathrm{NPCl}(\mathcal{A})$ implies $\mathcal{B} \quad \in \mathrm{NPGR} \alpha \mathrm{C}(\mathcal{J})$. 
Research Article

Proof: Let $\mathcal{B} \subseteq \mathcal{U}$ and $\mathcal{U}$ is a Neutrosophic regular $\alpha$-open set in $(\mathcal{J}, \tau)$. Since $\subseteq \mathcal{B}$, then $\mathcal{A} \subseteq \mathcal{U}$.Given $\mathcal{A}$ is a NPGR $\alpha \mathrm{CS}$, it follows that $\operatorname{NPCl}(\mathcal{A}) \subseteq \mathcal{U}$. Now $\mathcal{B} \subseteq \operatorname{NPCl}(\mathcal{A})$ implies $\operatorname{NPCl}(\mathcal{B}) \subseteq \operatorname{NPCl}(\operatorname{NPCl}(\mathcal{A}))=$ $\operatorname{NPCl}(\mathcal{A})$. Thus, $\operatorname{NPCl}(\mathcal{B}) \subseteq \mathcal{U}$. This proves that $\mathcal{B} \in \operatorname{NPGR} \alpha \mathrm{C}(\mathcal{J})$.

Theorem 2.12. If $\mathcal{A}$ is a Neutrosophic regular $\alpha$-open set and $\mathcal{N} \mathcal{P} \mathcal{G} \alpha \mathrm{CS}$ in $(\mathcal{J}, \quad \tau)$, then $\mathcal{A}$ is a Neutrosophic pre closed set in $(\mathcal{J}, \tau)$.

Proof: Since $\mathcal{A} \subseteq \mathcal{A}$ and $\mathcal{A}$ is a Neutrosophic regular $\alpha$-open set in $(\mathcal{J}$, $\tau)$, by hypothesis, $\mathrm{NPCl}(\mathcal{A}) \subseteq$ $\mathcal{A}$. But since $\mathcal{A} \subseteq \operatorname{NPCl}(\mathcal{A})$. Therefore $\operatorname{NPCl}(\mathcal{A})=\mathcal{A}$. Hence $\mathcal{A}$ is a Neutrosophic pre closed set in $(\mathcal{J}$, $\tau)$.

\section{NEUTROSOPHIC PRE GENERALIZED REGULAR $\alpha$-CLOSURE IN NEUTROSOPHIC TOPOLOGICAL} SPACES

In this section, we introduce the concept of Neutrosophic pre generalized regular $\alpha$-closure operators in a Neutrosophic topological spaces.

Definition 3.1. Let $(\mathcal{J}, \tau)$ be a Neutrosophic topological space. Then for a Neutrosophic subset $\mathcal{A}$ of $\mathcal{J}$,

(i) Neutrosophic pre generalized regular $\alpha$-interior of $\mathcal{A}$ is the union of all Neutrosophic pre generalized regular $\alpha$-open sets of $\mathcal{J}$ contained in $\mathcal{A}$. i.e., $\mathcal{N} \mathcal{P} \mathcal{G} \mathcal{R} \alpha-\operatorname{Int}(\mathcal{A})=\cup\{\mathcal{G}: \mathcal{G}$ is a $\mathcal{N} \mathcal{P} \mathcal{G} \mathcal{R} \alpha$-open set in $\mathcal{J}$ and $\mathcal{G}$ $\subseteq \mathcal{A}\}$.

(ii) Neutrosophic pre generalized regular $\alpha$-closure of $\mathcal{A}$ is the intersection of all Neutrosophic pre generalized regular $\alpha$-closed sets of $\mathcal{J}$ containing in $\mathcal{A}$. i.e., $\mathcal{N} \mathcal{P} \mathcal{G} \mathcal{R} \alpha-\operatorname{Cl}(\mathcal{A})=\bigcap\{\mathcal{K}: \mathcal{K}$ is a $\mathcal{N} \mathcal{P} \mathcal{G} \mathcal{R}$-closed set in $\mathcal{J}$ and $\mathcal{K} \supseteq \mathcal{A}\}$.

Theorem 3.2. Let $(\mathcal{J}, \tau)$ be a Neutrosophic topological space. Then for a Neutrosophic subsets $\mathcal{A}$ and $\mathcal{B}$ of $\mathcal{J}$, we have

(i) $\quad \mathcal{N} \mathcal{P} \mathcal{G} \mathcal{R} \alpha-\operatorname{Int}(\mathcal{A}) \subseteq \mathcal{A}$

(ii) $\quad \mathcal{A}$ is $\mathcal{N} \mathcal{P} \mathcal{G} \mathcal{R} \alpha$-open set in $\mathrm{A} \Leftrightarrow \mathcal{N} \mathcal{P} \mathcal{G} \mathcal{R} \alpha-\operatorname{Int}(\mathcal{A})=\mathcal{A}$

(iii) $\quad \mathcal{N} \mathcal{P} \mathcal{G} R \quad-\operatorname{Int}(\mathcal{N} \mathcal{P} \mathcal{G} \mathcal{R} \alpha-\operatorname{Int}(\mathcal{A}))=\mathcal{N} \mathcal{P} \mathcal{G} \mathcal{R} \alpha \quad-\operatorname{Int}(\mathcal{A})$

(iv) If $\mathcal{A} \subseteq \mathcal{B}$ then $\mathcal{N} \mathcal{P} \mathcal{G} R \quad-\operatorname{Int}(\mathcal{A}) \subseteq \quad \mathcal{N} \mathcal{P} \mathcal{G} R \quad-\operatorname{Int}(\mathcal{B})$

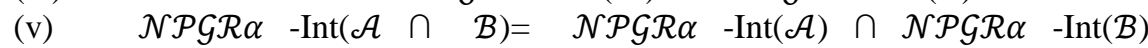

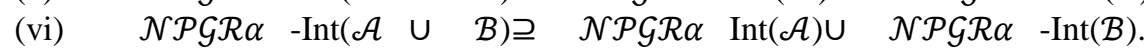

Proof: Follows from Definition 3.1.(i). This proves (i).

Let $\mathcal{A}$ be an $\mathcal{N} \mathcal{P} \mathcal{G} \mathcal{R} \alpha$-open set in $\mathcal{J}$. Then $\mathcal{A} \subseteq \mathcal{N} \mathcal{P} \mathcal{G} \mathcal{R} \alpha-\operatorname{Int}(\mathcal{A})$. By using Theorem 3.2 (i) we get $\mathcal{A}=\mathcal{N} \mathcal{P} \mathcal{G} \mathcal{R} \alpha-\operatorname{Int}(\mathcal{A})$. Conversely assume that $\mathcal{A}=\mathcal{N} \mathcal{P} \mathcal{G} \mathcal{R} \alpha-\operatorname{Int}(\mathcal{A})$. By using Definition $3.1(\mathrm{i}), \mathcal{A}$ is $\mathcal{N} \mathcal{P} \mathcal{G} \mathcal{R} \alpha-$ open set in $\mathcal{J}$. Thus (ii) is proved.

By using Theorem 3.2 (ii), $\mathcal{N} \mathcal{P} \mathcal{G} \mathcal{R} \alpha-\operatorname{Int}(\mathcal{N} \mathcal{P} \mathcal{G} \mathcal{R} \alpha-\operatorname{Int}(\mathcal{A}))=\mathcal{N} \mathcal{P} \mathcal{G} \mathcal{R} \alpha-\operatorname{Int}(\mathcal{A})$. This proves (iii).

Since $\mathcal{A} \subseteq \mathcal{B}$, by using Theorem 3.2 (i), $\mathcal{N} \mathcal{P} \mathcal{G} \mathcal{R} \alpha-\operatorname{Int}(\mathcal{A}) \subseteq \quad \mathcal{A} \subseteq \mathcal{B}$. That is $\mathcal{N} \mathcal{P} \mathcal{G} \alpha$-Int $(\mathcal{A}) \subseteq \quad \mathcal{B}$.

By Theorem 3.2 (iii), $\mathcal{N} \mathcal{P} \mathcal{G} \mathcal{R} \alpha-\operatorname{Int}(\mathcal{N} \mathcal{P} \mathcal{G} \mathcal{R} \alpha-\operatorname{Int}(\mathcal{A})) \subseteq \mathcal{N} \mathcal{P} \mathcal{G} \mathcal{R} \alpha-\operatorname{Int}(\mathcal{B})$. This proves (iv).

Since $\mathcal{A} \cap \mathcal{B} \subseteq \mathcal{A}$ and $\mathcal{A} \cap \mathcal{B} \subseteq \mathcal{B}$, by using Theorem 3.2(iv), $\mathcal{N} \mathcal{P} \mathcal{G} \mathcal{R} \alpha-\operatorname{Int}(\mathcal{A} \cap \quad \mathcal{B}) \subseteq \mathcal{N} \mathcal{P} \mathcal{G} \mathcal{R} \alpha-$ $\operatorname{Int}(\mathcal{A})$ and $\mathcal{N} \mathcal{P} \mathcal{G} \mathcal{R} \alpha-\operatorname{Int}(\mathcal{A} \cap \mathcal{B}) \subseteq \mathcal{N} \mathcal{P} \mathcal{G} \mathcal{R} \alpha-\operatorname{Int}(\mathcal{B})$.This implies that

$\mathcal{N} \mathcal{P} \mathcal{G} R-\operatorname{Int}(\mathcal{A} \quad \cap \quad \mathcal{B} \quad) \quad \subseteq \quad \mathcal{N} \mathcal{P} \mathcal{G} \mathcal{R} \alpha-\operatorname{Int}(\mathcal{A}) \quad \cap \quad \mathcal{N} \mathcal{P} \mathcal{G} \mathcal{R} \alpha-\operatorname{Int}(\mathcal{B})$

(1)

By using Theorem 3.2(i), $\mathcal{N} \mathcal{P} \mathcal{G} \mathcal{R} \alpha-\operatorname{Int}(\mathcal{A}) \subseteq \mathcal{A}$ and $\mathcal{N} \mathcal{P} \mathcal{G} \mathcal{R} \alpha-\operatorname{Int}(\mathcal{B}) \subseteq \mathcal{B}$. This implies that $\mathcal{N} \mathcal{P} \mathcal{G} \mathcal{R} \alpha-\operatorname{Int}(\mathcal{A}) \cap \mathcal{N} \mathcal{P} \mathcal{G} \mathcal{R} \alpha-\operatorname{Int}(\mathcal{B}) \subseteq(\mathcal{A} \cap \mathcal{B})$. Now applying Theorem 3.2(iv),

$\mathcal{N} \mathcal{P} \mathcal{G} R-\operatorname{Int}((\mathcal{N} \mathcal{P} \mathcal{G} \mathcal{R} \alpha-\operatorname{Int}(\mathcal{A}) \cap \mathcal{N} \mathcal{P} \mathcal{G} \mathcal{R} \alpha-\operatorname{Int}(\mathcal{B})) \subseteq \mathcal{N} \mathcal{P} \mathcal{G} \mathcal{R} \alpha-\operatorname{Int}(\mathcal{A} \cap \quad \mathcal{B} \quad)$. By Theorem 3.2(iii),

$\mathcal{N} \mathcal{P} \mathcal{G} \mathcal{R} \alpha-\operatorname{Int}(\mathcal{A}) \quad \cap \quad \mathcal{N} \mathcal{P} \mathcal{G} \mathcal{R} \alpha-\operatorname{Int}(\mathcal{B}) \quad \subseteq \mathcal{N} \mathcal{P} \mathcal{G} \mathcal{R} \alpha-\operatorname{Int}(\mathcal{A} \quad \cap \quad \mathcal{B} \quad)$

From equations (1) and (2), $\mathcal{N} \mathcal{P} \mathcal{G} \mathcal{R} \alpha-\operatorname{Int}(\mathcal{A} \cap \quad \mathcal{B})=\mathcal{N} \mathcal{P} \mathcal{G} \mathcal{R} \alpha-\operatorname{Int}(\mathcal{A}) \cap \mathcal{N} \mathcal{P} \mathcal{G} \mathcal{R} \alpha$-Int $(\mathcal{B})$. This $\operatorname{proves} \quad$ (v).

Since $\mathcal{A} \subseteq \mathcal{A} \cup \mathcal{B}$ and $\mathrm{B} \subseteq \mathcal{A} \cup \mathcal{B}$, by using Theorem 3.2(iv), $\mathcal{N} \mathcal{P} \mathcal{G} \alpha$-Int $(\mathcal{A}) \subseteq \mathcal{N} \mathcal{P} \mathcal{G} \alpha$-Int $(\mathcal{A} \cup \quad \mathcal{B})$

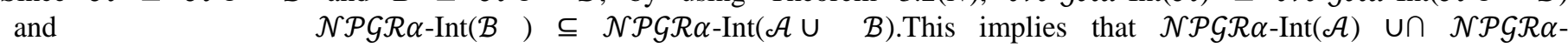
$\operatorname{Int}(\mathcal{B}) \subseteq \mathcal{N} \mathcal{P} \mathcal{G} \mathcal{R} \alpha-\operatorname{Int}(\mathcal{A} \cup \quad \mathcal{B})$. This proves (vi).

Remark 3.3. The following example shows that the equality need not hold in Theorem 3.3(vi).

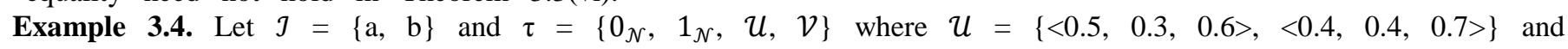
$\mathcal{V}=\left\{\begin{array}{lll}\langle 0.7, \quad 0.5,0.3\rangle,\langle 0.7,0.5,0.2\rangle\end{array}\right]$. Then $(\mathcal{J}, \tau)$ is a Neutrosophic topological

spaces. Consider the NSs

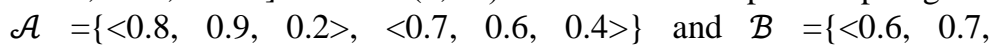
$0.5>,<0.9,0.8,0.2>\}$ in $(\mathcal{J}, \tau)$.

Then $\quad \mathcal{N} \mathcal{P} \mathcal{G} \mathcal{R} \alpha-\operatorname{Int}(\mathcal{A})=\left\{\begin{array}{lll}\langle 0.7, & 0.9, & 0.2 .>\end{array}\right), \quad<0.7, \quad 0.6$ 


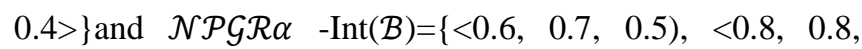

$0.2>$ \}, this implies $\mathcal{N} \mathcal{P} \mathcal{G} \mathcal{R} \alpha \quad-\operatorname{Int}(\mathcal{A}) \cup \quad \mathcal{N} \mathcal{P} \mathcal{G} \mathcal{R} \alpha-$

$\operatorname{Int}(\mathcal{B})=\left\{\begin{array}{llll}\langle 0.7,0.9, & 0.2\rangle,\langle 0.8,0.8,0.2\rangle\end{array}\right]$ But $\mathcal{N} \mathcal{P} G \mathcal{R} \alpha-\operatorname{Int}(\mathcal{A} \cup \mathcal{B})=\{\langle 0.8,0.9,0.2\rangle, \quad\langle 0.9,0.8,0.2\rangle\}$.

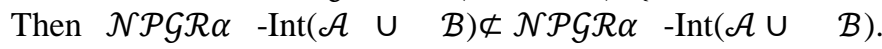

Proposition 3.5. Let $(\mathcal{J}, \tau)$ be a Neutrosophic topological space. Then for any Neutrosophic subsets $\mathcal{A}$ of $\mathcal{J}$,

(i) $\quad \mathrm{C}(\mathcal{N} \mathcal{P} \mathcal{G} \mathcal{R} \alpha-\operatorname{Int}(\mathcal{A}))=\mathcal{N} \mathcal{P} \mathcal{G} \mathcal{R} \alpha-\mathrm{Cl}(\mathrm{C}(\mathcal{A}))$,

(ii) $\quad \mathrm{C}(\mathcal{N} \mathcal{P} \mathcal{G} \mathcal{R} \alpha-\mathrm{Cl}(\mathcal{A}))=\mathcal{N} \mathcal{P} \mathcal{G} \mathcal{R} \alpha-\operatorname{Int}(\mathrm{C}(\mathcal{A}))$.

Proof : By using Definition 3.1(i), $\mathcal{N} \mathcal{P} \mathcal{G} \mathcal{R} \alpha-\operatorname{Int}(\mathcal{A})=$

$\cup\{\mathcal{G}: \mathcal{G}$ is a $\mathcal{N} \mathcal{P} \mathcal{G} \mathcal{R} \alpha$-open set in $\mathrm{A}$ and $\mathcal{G} \subseteq \mathcal{A}\}$.

Taking complement on both sides, $\mathrm{C}(\mathcal{N} \mathcal{P} \mathcal{G} \mathcal{R} \alpha-\operatorname{Int}(\mathcal{A}))=\mathrm{C}(\cup\{\mathcal{G}: \quad \mathcal{G}$ is a $\mathcal{N} \mathcal{P} \mathcal{G} \mathcal{R} \alpha$-open set in $\mathcal{J}$ and $\mathcal{G} \subseteq \mathcal{A}$ \}$)=\bigcap\{\mathrm{C}(\mathcal{G}): \mathrm{C}(\mathcal{G})$ is a $\mathcal{N} \mathcal{P} \mathcal{G} \mathcal{R} \alpha$-closed set in $\mathcal{J}$ and $\mathrm{C}(\mathcal{A}) \subseteq \mathrm{C}(\mathrm{G})\}$. Replacing $\mathrm{C}(\mathrm{G})$ by $\mathcal{K}$, we get $\mathrm{C}(\mathcal{N} \mathcal{P} \mathcal{G} \mathcal{R} \alpha-\operatorname{Int}(\mathcal{A}))=\cap\{\mathcal{K}: \quad \mathcal{K}$ is a $\mathcal{N} \mathcal{P} \mathcal{G} \mathcal{R} \alpha$-closed set in $\mathcal{J}$ and $\mathcal{K} \supseteq \mathrm{C}(\mathcal{A})\}$. By Definition $3.1(\mathrm{ii})$, $\mathrm{C}(\mathcal{N} \mathcal{P} \mathcal{G} \mathcal{R} \alpha-\operatorname{Int}(\mathcal{A}))=\mathcal{N} \mathcal{P} \mathcal{G} \mathcal{R} \alpha-\mathrm{Cl}(\mathrm{C}(\mathcal{A}))$. This proves (i).

By using Proposition 3.5 (i), $\mathrm{C}(\mathcal{N} \mathcal{P} \mathcal{G} \mathcal{R} \alpha-\operatorname{Int}(\mathrm{C}(\mathcal{A})))=\mathcal{N} \mathcal{P} \mathcal{G} \mathcal{R} \alpha-\mathrm{Cl}(\mathrm{C}(\mathrm{C}(\mathcal{A})))=\mathcal{N} \mathcal{P} \mathcal{G} \mathcal{R} \alpha-\mathrm{Cl}(\mathcal{A})$. on both sides, we get $\mathcal{N} \mathcal{P} \mathcal{G} \mathcal{R} \alpha-\operatorname{Int}(\mathrm{C}(\mathcal{A}))=\mathrm{C}(\mathcal{N} \mathcal{P} \mathcal{G} \mathcal{R} \alpha-\mathrm{Cl}(\mathcal{A}))$. Thus (ii) is proved.

Proposition 3.6. Let $(\mathcal{J}, \tau)$ be a Neutrosophic topological spaces. Then for any Neutrosophic subsets $\mathcal{A}$ and $\mathcal{B}$ of $\mathcal{J}$ we have

(i) $\quad \mathcal{A} \subseteq \mathcal{N} \mathcal{P} \mathcal{G} \mathcal{R} \alpha-\mathrm{Cl}(\mathcal{A})$.

(ii) $\quad \mathcal{A}$ is $\mathcal{N} \mathcal{P} \mathcal{G} \mathcal{R} \alpha$-closed set in $\mathcal{J} \Leftrightarrow \mathcal{N} \mathcal{P} \mathcal{G} \alpha-\operatorname{Cl}(\mathcal{A})=\mathcal{A}$.

(iii) $\quad \mathcal{N} \mathcal{P} \mathcal{G} R-\operatorname{Cl}(\mathcal{N} \mathcal{P} \mathcal{G} \alpha-\operatorname{Cl}(\mathcal{A}))=\mathcal{N} \mathcal{P} \mathcal{G} R-\operatorname{Cl}(\mathcal{A})$.

(iv) If $\mathcal{A} \subseteq \mathcal{B}$ then $\mathcal{N} \mathcal{P} \mathcal{G} \mathcal{R} \alpha-\mathrm{Cl}(\mathcal{A}) \subseteq \mathcal{N} \mathcal{P} \mathcal{G} \mathcal{R} \alpha-\mathrm{Cl}(\mathcal{B})$

Proof: Follows from the Definition 3.1(ii). This proves (i).

Let $\mathcal{A}$ be $\mathcal{N} \mathcal{P} \mathcal{G} \mathcal{R} \alpha$-closed set in J. Then $\mathrm{C}(\mathcal{A})$ is $\mathcal{N} \mathcal{P} \mathcal{G} \mathcal{R} \alpha$-open set in $\mathcal{J}$. By theorem 3.2(ii),

$\mathcal{N} \mathcal{P} G \mathcal{R} \alpha-$

$\operatorname{Int}(\mathrm{C}(\mathcal{A}))=\mathrm{C}(\quad \mathcal{A})$

$\Leftrightarrow \mathrm{C}(\mathcal{N} \mathcal{P} \mathcal{G} \mathcal{R} \alpha \mathrm{Cl}(\mathcal{A}))=\mathrm{C}(\mathcal{A}) \Leftrightarrow$

$\mathcal{N} \mathcal{P} \mathcal{G} R-\operatorname{Cl}(\mathcal{A})=\mathcal{A}$.Thus (ii) is proved.

By using Proposition 3.6 (ii), $\mathcal{N} \mathcal{P} \mathcal{G} \mathcal{R} \alpha-\mathrm{Cl}(\mathcal{N} \mathcal{P} \mathcal{G} \mathcal{R} \alpha-\mathrm{Cl}(\mathcal{A}))=\mathcal{N} \mathcal{P} \mathcal{G} \mathcal{R} \alpha-\mathrm{Cl}(\mathcal{A})$.This proves(iii).

Since $\quad \mathcal{A} \subseteq \mathcal{B}, \quad \mathrm{C}(\mathcal{B}) \subseteq \mathrm{C}(\mathcal{A})$. By using Theorem 3.2(iv), $\mathcal{N} \mathcal{P} \mathcal{G} \mathcal{R} \alpha$-Int $(\mathrm{C}(\mathcal{B})) \subseteq \mathcal{N} \mathcal{P} \mathcal{G} \mathcal{R} \alpha-\operatorname{Int}(\mathrm{C}(\mathcal{A}))$. Taking complement on both sides, $\quad \mathrm{C}(\mathcal{N} \mathcal{P} \mathcal{G} \mathcal{R} \alpha-\operatorname{Int}(\mathrm{C}(\mathcal{B}))) \supseteq \mathrm{C}(\mathcal{N} \mathcal{P} \mathcal{G} \mathcal{R} \alpha-\operatorname{Int}(\mathrm{C}(\mathcal{A})))$. By Proposition $3.5(\mathrm{ii}), \mathcal{N} \mathcal{P} \mathcal{G} \mathcal{R} \alpha-$ $\mathrm{Cl}(\mathcal{A}) \subseteq \mathcal{N} \mathcal{P} \mathcal{G} \mathcal{R} \alpha-\mathrm{Cl}(\mathcal{B})$. This proves(iv).

Proposition 3.7. Let $(\mathcal{J}, \tau)$ be a Neutrosophic topological spaces. Then for any Neutrosophic subset $\mathcal{A}$ and $\mathcal{B}$ of $\mathcal{J}$, we have

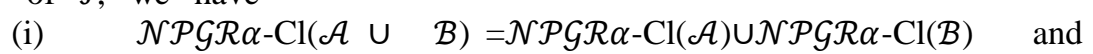

(ii) $\quad \mathcal{N} \mathcal{P} \mathcal{G} \mathcal{R} \alpha-\operatorname{Cl}(\mathcal{A} \cap \mathcal{B}) \subseteq \mathcal{N} \mathcal{P} \mathcal{G} \mathcal{R} \alpha-\operatorname{Cl}(\mathcal{A}) \cap \mathcal{N} \mathcal{P} \mathcal{G} \mathcal{R} \alpha-\operatorname{Cl}(\mathcal{B})$

Proof: Since $\mathcal{N} \mathcal{P} \mathcal{G} \mathcal{R} \alpha-\mathrm{Cl}(\mathcal{A} \cup \mathcal{B})=\mathcal{N} \mathcal{P} \mathcal{G} \mathcal{R} \alpha-\mathrm{Cl}(\mathrm{C}(\mathrm{C}(\mathcal{A} \cup \mathcal{B})))$, By using Proposition 3.5(i),

$\mathcal{N} \mathcal{P} \mathcal{G} R-\mathrm{Cl}(\mathcal{A} \cup \quad \mathcal{B})=\mathrm{C}(\mathcal{N} \mathcal{P} \mathcal{G} \mathcal{R} \alpha-\operatorname{Int}(\mathrm{C}(\mathcal{A} \cup \quad \mathcal{B})))=\mathrm{C}(\mathcal{N} \mathcal{P} \mathcal{G} \mathcal{R} \alpha-\operatorname{Int}(\mathrm{C}(\mathcal{A}) \cap \mathrm{C}(\mathcal{B})))$. Again using Proposition 3.2(v), $\mathcal{N} \mathcal{P} \mathcal{G} \mathcal{R} \alpha-\mathrm{Cl}(\mathcal{A} \cup \quad \mathcal{B})=\mathrm{C}(\mathcal{N} \mathcal{P} \mathcal{G} \mathcal{R} \alpha-\operatorname{Int}(\mathrm{C}(\mathcal{A})) \cap \mathcal{N} \mathcal{P} \mathcal{G} \mathcal{R} \alpha-\operatorname{Int}(\mathrm{C}(\mathcal{B})))=\mathrm{C}(\mathcal{N} \mathcal{P} \mathcal{G} \mathcal{R} \alpha-\operatorname{Int}(\mathrm{C}(\mathcal{A}))) \cup \mathrm{C}(\mathcal{N} \mathcal{P} \mathcal{G} \mathcal{R} \alpha-\operatorname{Int}(\mathrm{C}(\mathcal{B}))) \cdot \mathrm{By}$ using Proposition 3.5(i),

$\mathcal{N} \mathcal{P} \mathcal{G} R \alpha-\mathrm{Cl}(\mathcal{A} \quad \cup \quad \mathcal{B})=\mathcal{N} \mathcal{P} \mathcal{G} R-\mathrm{Cl} \quad(\mathrm{C}(\mathrm{C}(\mathcal{A}))) \cup \quad \mathcal{N} \mathcal{P} \mathcal{G} \mathcal{R} \alpha-\mathrm{Cl}(\mathrm{C}(\mathrm{C}(\mathcal{B})))=\mathcal{N} \mathcal{P} \mathcal{G} \mathcal{R} \alpha-\mathrm{Cl} \quad(\mathcal{A}) \cup \quad \mathcal{N} \mathcal{P} \mathcal{G} \alpha-\mathrm{Cl}(\mathcal{B})$

Thus (i) is proved.

Since $\mathcal{A} \cap \mathcal{B} \subseteq \mathcal{A}$ and $\mathcal{A} \cap \mathcal{B} \subseteq \mathcal{B}$, by using Proposition 3.6(iv), $\mathcal{N} \mathcal{P} \mathcal{G} \mathcal{R} \alpha-\mathrm{Cl}(\mathcal{A} \quad \cap \quad \mathcal{B}) \subseteq \mathcal{N} \mathcal{P} \mathcal{G} \mathcal{R} \alpha-$ $\mathrm{Cl}(\mathcal{A})$ and $\mathcal{N} \mathcal{P} \mathcal{G} \mathcal{R} \alpha-\mathrm{Cl}(\mathcal{A} \cap \quad \mathcal{B}) \subseteq \mathcal{N} \mathcal{P} \mathcal{G} \mathcal{R} \alpha-\mathrm{Cl}(\mathcal{B})$.

This implies that $\mathcal{N} \mathcal{P} \mathcal{G} \mathcal{R} \alpha-\operatorname{Cl}(\mathcal{A} \cap \quad \mathcal{B}) \subseteq \mathcal{N} \mathcal{P} \mathcal{G} \mathcal{R} \alpha-\operatorname{Cl}(\mathcal{A}) \cap \mathcal{N} \mathcal{P} \mathcal{G} \mathcal{R} \alpha-\mathrm{Cl}(\mathcal{B})$. This proves (ii).

Remark 3.8. The following example shows that the equality need not hold in Proposition 3.7(ii).

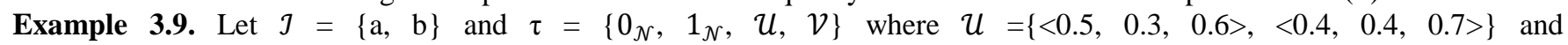
$\mathcal{V}=\{\langle 0.7,0.5,0.3\rangle,\langle 0.7,0.5,0.2\rangle\}$. Then $(\mathcal{J}, \tau)$ is a Neutrosophic topological space. Consider the NSs $\mathcal{A}=\{\langle 0.4,0.5,0.4>,\langle 0.7,0.6,0.8>\}$ and $\mathcal{B}=\{\langle 0.6,0.3,0.6\rangle,\langle 0.2,0.4,0.5\rangle\}$ in $(\mathcal{J}, \tau)$.

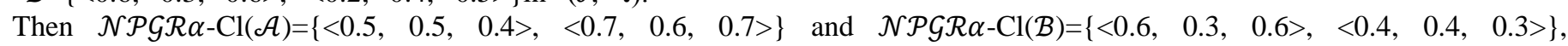

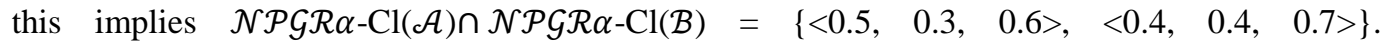

But $\mathcal{N} \mathcal{P} \mathcal{G} \mathcal{R} \alpha-\mathrm{Cl}(\mathcal{A} \cap \mathcal{B})=\{\langle 0.4,0.3,0.6\rangle, \quad\langle 0.2,0.4,0.8\rangle\}$.

Then $\mathcal{N} \mathcal{P} \mathcal{G} \mathcal{R} \alpha-\mathrm{Cl}(\mathcal{A}) \cap \mathcal{N} \mathcal{P} \mathcal{G} \mathcal{R} \alpha-\mathrm{Cl}(\mathcal{B}) \not \subset \mathcal{N} \mathcal{P} \mathcal{G} \mathcal{R} \alpha \quad \mathrm{Cl}(\mathcal{A} \quad \cap \mathcal{B})$. 
Remark 3.10. Let $\mathcal{A}$ be a Neutrosophic set in a Neutrosophic topological space on $\mathcal{J}$.

Then $\operatorname{NInt}(\mathcal{A}) \subseteq \mathcal{N} \mathcal{P} \mathcal{G} \mathcal{R} \alpha-\operatorname{Int}(\mathcal{A}) \subseteq \quad \mathcal{A} \subseteq \mathcal{N} \mathcal{P} \mathcal{G} \mathcal{R} \alpha-\operatorname{Cl}(\mathcal{A}) \subseteq \operatorname{NCl}(\mathcal{A})$.

Theorem 3.11. Let $(\mathcal{J}, \tau)$ be a NTS. Then for any

Neutrosophic subsets $\mathcal{A}$ and $\mathcal{B}$ of $\mathrm{A}$ we have

(i) $\quad \mathcal{N} \mathcal{P} G \mathcal{R} \alpha-\operatorname{Cl}(\mathcal{A}) \supseteq \quad \mathcal{A} \cup \mathcal{N} \mathcal{P} \mathcal{G} \mathcal{R} \alpha-\operatorname{Cl}(\mathcal{N} \mathcal{P} \mathcal{G} \alpha-\operatorname{Int}(\mathcal{A}))$.

(ii) $\quad \mathcal{N} \mathcal{P} \mathcal{G} \mathcal{R} \alpha-\operatorname{Int}(\mathcal{A}) \subseteq \quad \mathcal{A} \cap \mathcal{N} \mathcal{P} \mathcal{G} \mathcal{R} \alpha-\operatorname{Int}(\mathcal{N} \mathcal{P} \mathcal{G} \mathcal{R} \alpha-\operatorname{Cl}(\mathcal{A}))$

(iii) $\quad \operatorname{NInt}(\mathcal{N} \mathcal{P} \mathcal{G} R \alpha-\mathrm{Cl}(\mathcal{A})) \subseteq \operatorname{NInt}(\mathrm{NCl}(\mathcal{A}))$.

(iv) $\quad \operatorname{NInt}(\mathcal{N} \mathcal{P} \mathcal{G} \mathcal{R} \alpha-\operatorname{Cl}(\mathcal{A})) \supseteq \operatorname{NInt}(\mathcal{N} \mathcal{P} \mathcal{G} \mathcal{R} \alpha-\operatorname{Cl}(\mathcal{N} \mathcal{P} \mathcal{G} \mathcal{R} \alpha-\operatorname{Int}(\mathcal{A})))$

Proof:

By Proposition 3.6(i), $\mathcal{A} \subseteq \mathcal{N} \mathcal{P} \mathcal{G} \mathcal{R} \alpha-\mathrm{Cl}(\mathcal{A})$

Again using Theorem 3.2(i), $\mathcal{N} \mathcal{P} \mathcal{G} \mathcal{R} \alpha-\operatorname{Int}(\mathcal{A}) \subseteq \mathcal{A}$.

Then, $\mathcal{N} \mathcal{P} \mathcal{G} \mathcal{R} \alpha-\operatorname{Cl}(\mathcal{N} \mathcal{P} \mathcal{G} \mathcal{R} \alpha-\operatorname{Int}(\mathcal{A})) \subseteq \mathcal{N} \mathcal{P} \mathcal{G} \mathcal{R} \alpha-\operatorname{Cl}(\mathcal{A})$

(4).

$\operatorname{By}(3)$ and (4) we have, $\mathcal{A} \cup \mathcal{N} \mathcal{P} \mathcal{G} \mathcal{R} \alpha-\operatorname{Cl}(\mathcal{N} \mathcal{P} \mathcal{G} \alpha$ -

Int $(\mathcal{A})) \subseteq \mathcal{N} \mathcal{P} G \mathcal{R} \alpha-\operatorname{Cl}(\mathcal{A})$.

This proves (i).

Taking $\mathcal{N} \mathcal{P} \mathcal{G} \mathcal{R} \alpha$-interior on both sides of equation (3)

$\mathcal{N} \mathcal{P} \mathcal{G} \mathcal{R} \alpha-\operatorname{Int}(\mathcal{A}) \subseteq \mathcal{N} \mathcal{P} \mathcal{G} \mathcal{R} \alpha-\operatorname{Int}(\mathcal{N} \mathcal{P} \mathcal{G} \mathcal{R} \alpha-\mathrm{Cl}(\mathcal{A}))$

From (4) and (5), $\mathcal{N} \mathcal{P} \mathcal{G} \mathcal{R} \alpha-\operatorname{Int}(\mathcal{A}) \subseteq(\mathcal{A} \cap \mathcal{N} \mathcal{P} \mathcal{G} \mathcal{R} \alpha-$

$\operatorname{Int}(\mathcal{N} \mathcal{P} \mathcal{G} \mathcal{R} \alpha-\mathrm{Cl}(\mathcal{A})))$. This proves (ii).

By Remark 3.10, $\mathcal{N} \mathcal{P} \mathcal{G} \mathcal{R} \alpha-\mathrm{Cl}(\mathcal{A}) \subseteq \operatorname{NCl}(\mathcal{A})$. We get

This proves (iii).

$\operatorname{NInt}(\mathcal{N} \mathcal{P} \mathcal{G} \alpha-\operatorname{Cl}(\mathcal{A})) \subseteq \operatorname{NInt}(\mathrm{NCl}(\mathcal{A}))$.

By Theorem 3.11(i), $\mathcal{N} \mathcal{P} \mathcal{G} \mathcal{R} \alpha-\mathrm{Cl}(\mathcal{A}) \quad \supseteq(\mathcal{A} \quad \cup \quad \mathcal{N} \mathcal{P} \mathcal{G} \mathcal{R} \alpha-\mathrm{Cl}(\mathcal{N} \mathcal{P} \mathcal{G} \mathcal{R} \alpha-\operatorname{Int}(\mathcal{A})))$. Taking Neutrosophic interior on both sides, $\operatorname{NInt}(\mathcal{N} \mathcal{P} \mathcal{G} \mathcal{R} \alpha-\operatorname{Cl}(\mathcal{A}) \supseteq \operatorname{NInt}(\mathcal{A} \cup \mathcal{N} \mathcal{P} \mathcal{G} \mathcal{R} \alpha-\operatorname{Cl}(\mathcal{N} \mathcal{P} \mathcal{G} \mathcal{R} \alpha-\operatorname{Int}(\mathcal{A})))$.

Since $\quad \operatorname{NInt}(\mathcal{A} \cup \quad \mathcal{B}) \supseteq \operatorname{NInt}(\mathcal{A}) \cup \operatorname{NInt}(\mathcal{B}), \quad \operatorname{NInt}(\mathcal{N} \mathcal{P} \mathcal{G} \mathcal{R} \alpha-\operatorname{Cl}(\mathcal{A}) \supseteq \operatorname{NInt}(\mathrm{A}) \cup \operatorname{NInt}(\mathcal{N} \mathcal{P} \mathcal{G} \mathcal{R} \alpha-\operatorname{lnt}(\mathcal{N} \mathcal{P} \mathcal{G} \mathcal{R} \alpha-$ $\operatorname{Int}(\mathcal{A}))) \supseteq \operatorname{NInt}(\mathcal{N} \mathcal{P} \mathcal{G} \mathcal{R} \alpha-\operatorname{Cl}(\mathcal{N} \mathcal{P} \mathcal{G} \mathcal{R} \alpha-\operatorname{Int}(\mathcal{A})))$. Thus (iv) is proved.

Theorem 3.12.Let $(\mathcal{J}, \tau)$ be a Neutrosophic topological space. Then for any Neutrosophic subset $\mathcal{A}$ and $\mathcal{B}$ of $\mathcal{J}$

(i) $\quad \mathcal{N} \mathcal{P} \mathcal{G} \mathcal{R} \alpha-\mathrm{Cl}(\mathcal{A}) \supseteq \quad \mathcal{A} \cup \mathcal{N} \mathcal{P} \mathcal{G} \mathcal{R} \alpha-\operatorname{Cl}(\mathcal{N} \mathcal{P} \mathcal{G} R-\operatorname{Int}(\mathcal{A}))$

(ii) $\quad \mathcal{N} \mathcal{P} \mathcal{G} \mathcal{R} \alpha-\operatorname{Int}(\mathcal{A}) \subseteq \quad \mathcal{A} \cap \mathcal{N} \mathcal{P} \mathcal{G} \mathcal{R} \alpha-\operatorname{Int}(\mathcal{N} \mathcal{P} \mathcal{G} \alpha-\operatorname{Cl}(\mathcal{A}))$.

(iii) $\quad \operatorname{NInt}(\mathcal{N P} \mathcal{P} \mathcal{R} \alpha-\mathrm{Cl}(\mathcal{A})) \subseteq \operatorname{NInt}(\operatorname{NCl}(\mathcal{A}))$.

(iv) $\quad \operatorname{NInt}(\mathcal{N} \mathcal{P} \mathcal{G} \mathcal{R} \alpha-\mathrm{Cl}(\mathcal{A})) \supseteq \operatorname{NInt}(\mathcal{N} \mathcal{P} \mathcal{G} \mathcal{R} \alpha-\mathrm{Cl} \quad(\mathcal{N} \mathcal{P} \mathcal{G} \mathcal{R} \alpha-\operatorname{Int}(\mathcal{A})))$.

Proof: By Proposition 3.6(i), $\mathcal{A} \subseteq \mathcal{N} \mathcal{P} \mathcal{G} \mathcal{R} \alpha-\mathrm{Cl}(\mathcal{A})$

Again using Theorem 3.2(i),

$\mathcal{N} \mathcal{P} G \mathcal{R} \alpha-\operatorname{Int}(\mathcal{A}) \subseteq \mathcal{A}$.

Taking $\mathcal{N} \mathcal{P} \mathcal{G} \mathcal{R} \alpha$-closure on both sides, $\mathcal{N} \mathcal{P} \mathcal{G} \mathcal{R} \alpha-\mathrm{Cl}(\mathcal{N} \mathcal{P} \mathcal{G} \mathcal{R} \alpha-\operatorname{Int}(\mathcal{A})) \subseteq \mathcal{N} \mathcal{P} \mathcal{G} \alpha-\mathrm{Cl}(\mathcal{A})$

(7).

By equation (6) and (8), $\mathcal{A} \cup \mathcal{N} \mathcal{P} \mathcal{G} \mathcal{R} \alpha-\operatorname{Cl}(\mathcal{N} \mathcal{P} \mathcal{G} \mathcal{R} \alpha-\operatorname{Int}(\mathcal{A})) \subseteq \mathcal{N} \mathcal{P} \mathcal{G} \mathcal{R} \alpha-\mathrm{Cl}(\mathcal{A})$.

This proves (i).

Again using Theorem 3.2(i), $\mathcal{A} \subseteq \mathcal{N} \mathcal{P} \mathcal{G} \mathcal{R} \alpha-\mathrm{Cl}(\mathcal{A})$. Taking $\mathcal{N} \mathcal{P} \mathcal{G} \mathcal{R} \alpha$-interior on both sides, $\mathcal{N} \mathcal{P} \mathcal{G} \mathcal{R} \alpha-\operatorname{Int}(\mathcal{A}) \subseteq \mathcal{N} \mathcal{P} \mathcal{G} \mathcal{R} \alpha-\operatorname{Int}(\mathcal{N} \mathcal{P} \mathcal{G} \mathcal{R} \alpha-\operatorname{Cl}(\mathcal{A}))$

(9).

From (7) and (9), we have $\mathcal{N} \mathcal{P} \mathcal{G} \mathcal{R} \alpha-\operatorname{Int}(\mathcal{A}) \subseteq(\quad \mathcal{A} \cap \mathcal{N} \mathcal{P} \mathcal{G} \mathcal{R} \alpha-\operatorname{Int}(\mathcal{N} \mathcal{P} \mathcal{G} \mathcal{R} \alpha-\mathrm{Cl}(\mathcal{A}))$ ). This proves (ii).

By Theorem 3.11, $\mathcal{N} \mathcal{P} \mathcal{G} \mathcal{R} \alpha-\mathrm{Cl}(\mathcal{A}) \subseteq \operatorname{NCl}(\mathcal{A})$. Then $\operatorname{NInt}(\mathcal{N} \mathcal{P} \mathcal{G} \mathcal{R} \alpha-\mathrm{Cl}(\mathcal{A})) \subseteq \operatorname{NInt}(\mathrm{NCl}(\mathcal{A})$ ). Thus (iii) is proved.

By Theorem 3.12(i), $\mathcal{N} \mathcal{P} \mathcal{G} \mathcal{R} \alpha-\operatorname{Cl}(\mathcal{A}) \supseteq(\quad \mathcal{A} \cup \mathcal{N} \mathcal{P} \mathcal{G} \mathcal{R} \alpha-(\mathcal{N} \mathcal{P} \mathcal{G} \mathcal{R} \alpha-\operatorname{Int}(\mathcal{A})))$.

This implies $\operatorname{NInt}(\mathcal{N} \mathcal{P} \mathcal{G} \mathcal{R} \alpha-\operatorname{Cl}(\mathcal{A}) \supseteq \operatorname{NInt}(\mathcal{A} \cup \mathcal{N} \mathcal{P} \mathcal{G} \mathcal{R} \alpha-\operatorname{Cl}(\mathcal{N} \mathcal{P} \mathcal{G} \mathcal{R} \alpha-\operatorname{Int}(\mathcal{A})))$.

Since $\quad \operatorname{NInt}(\mathcal{A} \cup \quad \mathcal{A}) \supseteq \operatorname{NInt}(\mathcal{A}) \cup \operatorname{NInt}(\mathcal{B}), \mathcal{N} \mathcal{P} \mathcal{G} \mathcal{R} \alpha-\operatorname{Int}(\mathcal{N} \mathcal{P} \mathcal{G} \mathcal{R} \alpha-\operatorname{Cl}(\mathcal{A}) \supseteq \operatorname{NInt}(\mathcal{A}) \cup \operatorname{NInt}(\mathcal{N} \mathcal{P} \mathcal{G} \alpha-\operatorname{Cl}(\mathcal{N} \mathcal{P} \mathcal{G} \mathcal{R} \alpha-$ $\operatorname{Int}(\mathcal{A}))) \supseteq \operatorname{NInt}(\mathcal{N} \mathcal{P} \mathcal{G} \mathcal{R} \alpha-\operatorname{Cl}(\mathcal{N} \mathcal{P} \mathcal{G} \mathcal{R} \alpha-\operatorname{Int}(\mathcal{A})))$. Thus (iv) is proved.

\section{REFERENCES}


[1] M.Abdel-Baset, M., Chang V., and Gamal A., Evaluation of the green supply chain management practices: A Novel neutrosophic approach, Computers in Industry, Vol. 108, pp.210-220, 2019.

[2] Abdel-Basset, M., El-hoseny, M., Gamal, A., \&Smarandache,

F. A novel model for evaluation Hospital medical care systems based on plithogenic sets. Artificial intelligence in medicine,

Vol.100, pp.101-110, 2019.

[3] Abdel-Basset, M., Mohamed, R., Zaied, A. E. N. H., \& marandache, F., A hybrid plithogenic decisionmaking approach with quality function deployment for selecting supply chain sustainability metrics, Symmetry,Vol.11(7), pp.90, 2019.

[4] Abdel-Basset, M., Saleh, M., Gamal, A., \&Smarandache, F. (2019). An approach of TOPSIS technique for developing supplier selection with group decision making under type-2 neutrosophic number, Applied Soft Computing, Vol.77, pp.438- 452, 2019.

[5] Atanassov K.T., Intuitionistic fuzzy sets, Fuzzy Sets and Systems, pp.87-96, 1986.

[6] Chang C. L., Fuzzy topological spaces J.Math.Anal.Appl. Vol.24,No.1, pp. 182- 190, 1968.

[7] D. Coker, An Introduction to Intuitionistic fuzzy topological spaces, Fuzzy Sets and Systems,Vol. 88, No.1,pp. 81-89, 1997.

[8] R.Dhavaseelan and S. Jafari, Generalized Neutrosophic closed sets, New trends in Neutrosophictheory and applications Vol. II, pp.261-273,2018.

[9] Harshitha A. and Jayanthi D., Regular a-Generalized Closed Ses in Neutrosophic topological Spaces, IOSR Journal of Mathematics, Vol.15,No. 02,pp.11-18, 2019.

[10] Ishwarya P.and K. Bageerathi, On Neutrosophic semi-open sets in Neutrosophic topological spaces,International Journal of math. Trends and Tech., pp.214-223, 2016.

[11] I.M.A. Jaffer and K. Ramesh, Neutrosophic Generalized Pre Regular Closed Sets, Neutrosophic Sets and Systems,Vol. 30, pp.171, 2019

[12] Jayanthi D., On $\alpha$ Generalized closed sets in Neutrosophic topological spaces, International conference on Recent trends in mathematics and Information technology (ICRMIT 2018)

[13] W. Al-Omeri and S. Jafari, On Generalized Closed Sets and Generalized pre-Closed sets in Neutrosophic Topological Spaces, Mathematics MDPI,Vol.7, No.1,pp. 01-12, 2018.

[14] W. Al-omeri and F. Smarandache, New Neutrosophic sets via Neutrosophic Topological spaces, New Trends in Neutrosophic Theory and Applications, Vol(2), June 2016.

[15] A.Pushpalatha and T. Nandhini, Generalized closed sets via neutrosophic topological spaces, Malaya Journal of Matematik,

Vol.7, No.1, pp.50-54, 2019

[16] Salama A.A. and Alblowi S.A., Neutrosophic set and Neutrosophic topological space, IOSR Journal of Mathematics, Vol.3, No.4, pp. 31-35,2012.

[17] Salama A. A., F.Smarandache and V. Kroumov, Neutrosophic closed set and Neutrosophic continuous functions, Neutrosophic Sets and Systems, Vol. 4,pp. 4-8,2014.

[18] Santhi R. and Udhayarani N.,et al., No -Closed sets in Neutrosophic Topological Spaces, Neutrosophic Sets and Systems, Vol.12,No.1, pp. 114-117, 2016.

[19] F. Smarandache ,Neutrosophic and Neutrosophic Logic, First International Conference on Neutrosophic ,Neutrosophic Logic,

Set, Probability, and Statistics, University of New Mexico,

[20]Zadeh, L.A., Fuzzy sets, Information and Control, Vol. 8,

Gallup, NM, Vol. 87301, USA (2002).

No.3, pp.338-353, 1965. 